[REVIEW]

\title{
The Syntax of Roots and the Roots of Syntax
}

Ed. by Artemis Alexiadou, Hagit Borer, and Florian Schäfer, Oxford University Press, Oxford, 2014, xiii+333pp.

JUNYA MORITA

Kinjo Gakuin University*

Keywords: antilexicalism, root, categorizing head, syntactic computation, idiosyncrasy

\section{Introduction}

Traditionally, roots are defined as morphemes that constitute the nuclei or cores of words; they are either free as in a cat and fix a mirror on the wall or bound as in wildcat and fixation (Nida (1949: 82-83)). In the current theory of antilexicalism, however, which situates major word formation within syntax, all roots are bound as in $\left[{ }_{n \mathrm{P}} n \sqrt{ }\right.$ cat $]$, consisting of the categorizing morpheme $n$ and the root $\sqrt{c a t}_{\text {cat }}$ The boundness of roots plays a critical role in word and sentence construction: the parallel argument structure of a VP and its nominal is attributable to the common root, major idiosyncrasies are limited to roots, and phase-based, step-by-step vocabulary insertion is carried out (cf. [np -ation [ $\mathrm{vp}_{\mathrm{P}}$-ize $[a \mathrm{P}-\varnothing \sqrt{ }$ modern] $\left.\left.]\right]\right)$. The essential task for antilexicalists is then to reveal how and to what extent roots play syntactic and semantic roles.

The main objective of the book under review is to undertake such a challenging task by elucidating different views of roots and their merits and defects. This book derives from two workshops held in 2009 and it

* I would like to express my gratitude to two anonymous referees for their valuable comments and suggestions on an earlier draft of this review. I am also indebted to Yuji Takano for providing me with a basic knowledge of the Minimalist Program. Special thanks are due to Matthew A. Taylor, who kindly corrected stylistic errors in an early version of this review. Needless to say, I am solely responsible for any remaining inadequacies. This work is partly supported by a Grant-in-Aid for Scientific Research (C) (No. 26370462) from the Japan Society for the Promotion of Science.

English Linguistics 34: 1 (2017) 81-92 -81-

(C) 2017 by the English Linguistic Society of Japan 
contains an introduction by the editors (Chapter 1) and twelve contributions (Chapters 2-13). Notably, this volume has four central questions, as explicitly indicated in Chapter 1: (i) is it roots or governing categories that play a central role in sentence construction?, (ii) how narrowly is the domain of idiosyncratic meanings defined?, (iii) how are category-neutral roots connected with syntactic categories?, and (iv) how are roots bound up with phases? Differing analyses for each of these questions are put forward in this book. The present review outlines these analyses one by one, raises specific questions for each system, and comments on its theoretical implications. For reasons of space, and because some chapters represent a similar position, not all chapters will be explicitly treated in this review. ${ }^{1}$

\section{Four Major Themes}

\subsection{Root-centered versus Category-centered}

\subsubsection{Outline}

As the title of the book implies, two opposing positions are recognizable on the syntactic role of roots: roots are crucially involved in constructing syntactic and semantic structures (position 1), and what is essential in sentence construction is the dominant categories of a root, in particular its head category (position 2). Position 2 is advocated by Acedo-Matellán and Mateu in Chapter 2 of the book, whose central claim is that a root is structurally interpreted drawing on the syntactic position it takes, arguing against the grammatical relevance of roots (root ontology). This approach has the merit of accounting for the complementary distribution of Manner/Result readings; break-verbs can be assigned a Result or Manner reading, as shown in (1) and (2) respectively, but they cannot bear both readings simultaneously (pp. 20-21).

(1) a. The strong winds broke the glass.

b. $\quad\left[{ }_{v \mathrm{P}}[\mathrm{DP}\right.$ the strong winds $]\left[v_{v^{\prime}} v\left[{ }_{\mathrm{PP}}\left[{ }_{\mathrm{DP}}\right.\right.\right.$ the glass $]\left[_{\mathrm{P}^{\prime}} \mathrm{P}\left[_{\mathrm{PP}} \mathrm{P}\right.\right.$ Vbreak]]]]]

(2) a. The hammer head broke off.

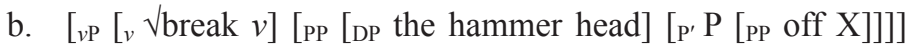

1 The principle arguments for the significance of root meaning are advanced in this volume by five of the leading experts in the field-Alexiadou (Chapter 4), Doron (Chapter 8), Levinson (Chapter 10), Rappaport Hovav (Chapter 12), and Roßdeutscher (Chapter 13). By taking Doron's chapter as representative of position 1, for reasons of space, our discussion will be confined to the arguments for the root ontology developed in Chapter 8 . 
Each reading is derived from the corresponding syntactic structure; in (1b) a root occupies the argument position in an event schema and consequently the Result reading of 'the glass became a state of being broken' is obtained. By contrast, a root occupies the place of a $v$-modifier in (2b), resulting in the Manner reading of 'the hammer head became separated from the hammer in a manner of breakage.' The root $\sqrt{ }$ break cannot appear in both places simultaneously, hence the complementarity of Manner and Result. The view that roots have no complements or specifiers and hence no projections of their own also deals well with the Manner/Result complementarity of cognate objects (pp. 21-22).

The root ontology (position 1), on the other hand, is supported by Doron in Chapter $8 .^{2}$ It is argued here that the information characterizing adjectival passives (APs) in Hebrew is located in roots. Verbal derivation of a template is schematized in (3), where detailed information on arguments and eventualities is included in the root $\sqrt{ } \mathrm{mkr}$ 'sell.' The semantic element $<$ Target $>$ ascribed to the root is important, since a target state is a necessary condition for AP formation in Hebrew. For this reason, the AP makur 'sold' is correctly derived, whereas the roots which do not imply a target state cannot generate APs, as evidenced in *daxup s 'pushed.'
(3) $\mathrm{z}$ makar et-X

$\mathrm{z}$ sell-SIMPL ACC- $\mathrm{x}$
'z sold $\mathrm{x}$ '

$\lambda z \lambda e$ [Agent $(e, z)]$

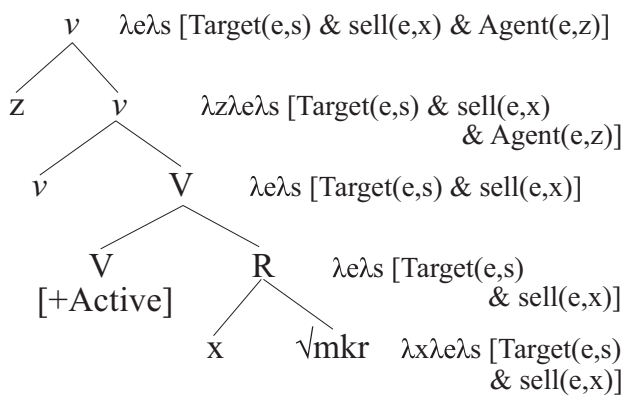

(p. 184)

\subsubsection{Evaluation}

We may say that which approach is preferred depends on the type of syntactic construction or pattern; the complementarity of Manner/Result readings cannot be adequately handled by position 1 , where it would be possible

\footnotetext{
2 See Note 1.
} 
to place in a root the semantic features which enable Manner/Result readings to coexist. Conversely, position 2 fails to deal properly with the necessary condition of <Target $>$ for AP formation in Hebrew; it would require some rather ad-hoc treatment which places <Target> in a functional head (P). It is therefore necessary to examine some other constructions from the above viewpoints before reaching any conclusion as to which is more appropriate.

Particularly worthy of comment are the theoretical implications of both positions for vocabulary insertion. One of the central claims of Distributed Morphology (DM) is late insertion: in the syntactic interfaces, vocabularyparticular phonological and semantic information is copied onto the relevant terminal nodes. This antilexicalist thesis has the merit of enforcing the economy principle that places severe restrictions on the kind of information available at each level (Halle (1994)); if vocabulary-particular phonological/ semantic information were distributed in syntax, it would be carried over to the PF and LF components without playing any role. In relation to late insertion, the two approaches have different consequences: position 1 claims that the semantic features of roots play a crucial role in syntactic construction and hence roots exist during syntactic computation, whereas position 2 considers roots only as providers of encyclopedic meaning, which is supplied in the C-I interface (p. 17). Thus, position 2 fits naturally into the late insertion thesis, whereas position 1 has to take into consideration some means of conforming itself to the antilexicalist thesis in order to hold relevant economical merit. ${ }^{3}$

\subsection{Non-compositionality Domain}

\subsubsection{Outline}

The second focal point is to identify ways in which non-compositional interpretations emerge and define the domain of non-compositionality. A generalization about this has been proposed by Marantz and Arad: a root is assigned an interpretation in the context of the first categorial head of the root, which is then retained throughout the derivation (p. 81). This hypothesis is closely examined in Chapters 5 and 7 of the book. It is argued

${ }^{3}$ It is currently suggested that roots are not subject to vocabulary insertion although functional morphemes are later inserted (Embick (2010)). However, this would entail that the bulk of vocabulary insertion not follow the late insertion thesis, thus discarding a relevant economical advantage. 
by Anagnostopoulou and Samioti in Chapter 5 that the hypothesis is too strong, while arguments in favor of it are provided by De Belder, Faust, and Lampitelli in Chapter 7.

Let us first outline the main arguments of Chapter 5. Greek has the verbal adjective-forming suffix -tos and adjectival participle-forming suffix -menos. The former does not imply events, indicating "inner-cycle attachment" (below $v \mathrm{P} /$ VoiceP), while the latter denotes states resulting from prior events, indicating "outer-cycle attachment" (above $v \mathrm{P} /$ VoiceP). Given the Marantz/Arad hypothesis, it is predicted that -tos may have idiosyncratic meanings, whereas -menos has only transparent meanings. However, this prediction is not always borne out. First, the "outer-attachment" -menos may take on a special meaning; the -menos participle stri-menos '(lit.) twisted' has a non-transparent reading in the nominal stri-menos geros 'crotchety old man' (p. 106). Secondly, a type of -tos may attach to "root + verbalizer," producing an unpredictable meaning (cf. [kol-i]-tos, glue-verbalizertos 'close friend') (p. 106). Thirdly, another type of -tos, which denotes ability/possibility (pistef-t-i, believe-t-fem.sg.nom 'believable'), can license both agent PPs and agent-oriented adverbs and accordingly it attaches above v. This type of -tos verbal adjective has only predictable meanings (p. 92).

Chapter 7 reveals the distinctive features of two kinds of diminutive (DIM) in Italian and Modern Hebrew and accounts for the origin of these features along the lines of Distributed Morphology. Semantically, type 1 DIM is exclusively compositional as in nas-ino (nose.DIM, 'small nose'), while type 2 DIM gives rise to non-compositional imports as in pan-ino (bread.DIM, 'sandwich') (p. 149). Morphologically, type 1 is fully productive while type 2 is unproductive; in addition, morphosyntactically, type 2 can associate with more than one category while type 1 selects just one category. These characteristics of the two DIMs follow straightforwardly from their occurrence in two different syntactic positions; type 1 occurs in the $\operatorname{Size}^{0}$ position and type 2 occupies the $\operatorname{Lex}^{0}$ position in the nominal

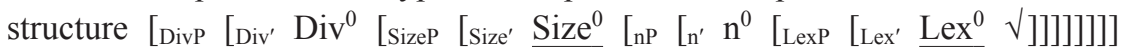
(p. 151). Assuming that the categorial head $n$ constitutes a boundary for "lexical" properties, the fact that type 2 DIM is readily associated with "irregularities"- semantic non-transparency, accidental gaps, and non-selection of the head-is well captured by the domain of type 2, that is, the domain below that head.

\subsubsection{Appraisal}

Before commenting on the two approaches, let us mention the theoretical 
background for the advent of the Marantz/Arad hypothesis. In a theory of antilexicalism, the syntactic structures of multiply affixed words are uniformly constructed by the merging of a root and category-defining heads without any distinction of affixes. ${ }^{4}$ As it stands, non-compositional interpretations would emerge from any constituent of the structure. Furthermore, currently local vocabulary insertion is proposed: allomorph selection is made in local environments by cyclic operations at PF (Embick (2010)), and likewise at LF "alloseme" choice for insertion is carried out in an inner-to-outer and phase-based manner (Marantz (2013)). In word structures with stacked $x$ heads, an outer head is not allowed to affect the spelled-out complement phonologically and semantically. In two respects, then, some means is required to define the scope for non-compositionality as narrowly as possible.

Attractive as it may seem, the Marantz/Arad hypothesis is too restrictive; (i) "outer attachment" morphemes may trigger lexicalized meanings, and (ii) a lower morpheme attaching to a root-head composite may produce non-compositionality, as discussed in Chapter 5. One way of coping with the second difficulty is to identify the head concerned as non-phasal. In the Greek participle kol-i-tos given above, the verbalizer $-i$ is semantically null and has no event implications. Since semantically -tos attaches directly to the root, the intervening $v$ is not itself a category head and it is accordingly ignored in demarcation of the non-compositionality domain (cf. Marantz (2013: 110-111)). A possible way of tackling the first issue is to divide affixes into two types along the lines suggested in Chapter 7. As the Italian diminutive -ino is subdivided into type 1 and type 2 DIMs, the Greek participial suffix -menos can be classified into two main types in terms of distinctive properties - compositionality, productivity, and structural site. For this approach to succeed, it will be necessary to explore the means by which to explain why affixes in the same form belong to different classes and why the distinctive properties of one type may not always cluster together. It may be worthwhile trying to apply a sort of "grammaticalization" view to the optimum linking of two types of affixes, as suggested in Chapter 7 (p. 162); non-compositional -menos can be extended from its compositional counterpart under certain narrowly definable conditions.

${ }^{4}$ For theoretical and empirical evidence in support of the antilexicalist view, see Marantz (1997), Harley and Noyer (2000), and Morita (2007), among others. 
2.3. Categorized Terminal versus Category-less Terminal

\subsubsection{Outline}

The third major issue of the book is how to associate category-neutral roots with syntactic categories. One approach is the contextual categorization (CC) model advanced by Borer in Chapter 6: the category is determined by the syntactic context in which a root occurs. In (4a) for instance, the $\mathrm{C}$-functor $\mathrm{C}_{\mathrm{N}[\mathrm{V}]}$ defines the outer spatial domain as $\mathrm{N}$ and the complement space as $V$, thereby specifying the root $V_{\text {form }}$ in the complement position as V. The other approach is adopted by the current framework of DM: categorization of roots is achieved only by the merging of roots and $x$ heads, as (4b) indicates.

(4)

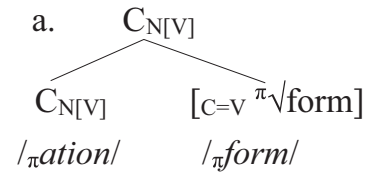

b.

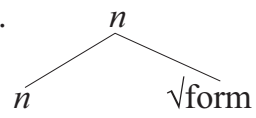

lation/ /form/

(cf. Embick (2010))

It should be stressed that the DM system makes null exponents inevitable;

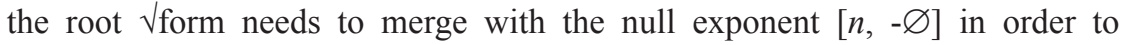
produce the noun form. By contrast, the $\mathrm{CC}$ analysis does not make zero morphemes inevitable; the noun forms takes the structure [CL DIV ${ }_{C L}[\mathrm{C}=\mathrm{N}$ $\pi \sqrt{\text { form }}]]$, where the functor $\left[\mathrm{DIV} \mathrm{CL}_{\mathrm{CL}},-\mathrm{s}\right]$ directly selects $\mathrm{N}$ without the intervention of $[n,-\varnothing]$ (pp. 115-116). Thus, the crucial difference between the two systems is that DM requires a root to remain a category-less terminal, thereby producing null categorizers as a logical consequence, whereas $\mathrm{CC}$ requires a root to become a categorized terminal.

\subsubsection{Assessment}

Borer's analysis in Chapter 6 is particularly valuable in distinguishing whether roots are always category-less terminals (DM) or categorized terminals (CC) and in refuting the former approach in a fairly straightforward way. She argues convincingly that the DM approach would suffer an unnecessary proliferation of $x$ heads, leading to a serious imbalance between underlying structures and their instantiations and a failure to lay down a key condition for the domain of compositionality. However, two questions can be raised regarding Borer's criticisms of the zero categories which concern $\mathrm{N}-\mathrm{V}$ alternation. First, claiming that derived nouns may not be converted into verbs as in *to salutation, the author derives this general restriction from the non-existence of null morphemes as C-functors ${ }^{*}[\mathrm{Cv}[\mathrm{N}]-\varnothing[\mathrm{CN}[\mathrm{V}]$ 


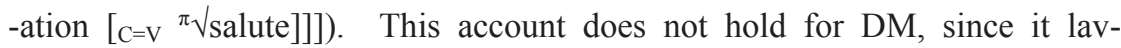
ishly makes use of zero categories. Yet, since the CC framework does not completely deny the existence of zero morphemes (pp. 121-122), the prohibition of zero realizations of $\mathrm{C}$-functors requires explanation. Additionally, the alleged restriction which prevents derived nouns from undergoing conversion is open to counter-examples as in partition $_{V}$ and proposition $_{V}$, and so a unified account of the restriction and its counter-examples will be required. Second, pointing out that zero-deverbal nouns have no argument structure, Borer argues that $\mathrm{CC}$ distinguishes argument-taking derived nominals ([CN[v] -ation $[\mathrm{C}=\mathrm{V} \pi \sqrt{ }$ export $]])$ from non-argument-taking zero-deverbal nominals $([\mathrm{C}=\mathrm{N} \pi \sqrt{ }$ export $])$ by their different syntactic configurations, whereas DM fails to make a clear distinction between the two nominals since they represent a common configuration, as in (4b) above. Nevertheless, "a large number of exceptions exist" (Roeper (1993: 216)) and there also exist some overt suffixes with a poor ability to inherit arguments (cf. *the scene's recurrence to him), indicating that argument inheritance may not be determined by the overt-covert differences of affixes. ${ }^{5}$

What should be expected of the CC view is for it to show how the entries of roots are represented and how vocabulary insertion is implemented. Regarding the semantic information of "conversions," DM explicitly shows that this is distributed to a root and functional categories; the meaning of the verb green ([ $v_{-\varnothing}\left[a_{-\varnothing}\left[n_{-\varnothing}\right.\right.$ V green $\left.\left.\left.]\right]\right]\right)$ is determined by combining the meaning of each item-the root and null morphemes (Embick (2010)). How then can the $\mathrm{CC}$ analysis treat this? Does the root Vgreen have submeanings such as 'color of grass,' 'having the color of grass,' and 'make (something) have the color of grass' to be presented in relevant contexts? And if so, would the root take on its original sense plus the senses of other elements? The licensing conditions on vocabulary insertion are also not clear: what permits the item related to green $_{V}$ to be inserted into the $\mathrm{V}$ node, and what disallows the insertion of the comparable item for *equal ${ }_{V}$ ?

\subsubsection{A Compromise Position}

We have so far identified two positions about how roots are categorized,

${ }^{5}$ Borer provides additional arguments against zero derivation (pp. 139-146), but they are consistently against the level distinction of zero morphemes, not against zero derivation itself. 
"categorization by syntactic context" and "categorization by the direct merge of functional categories," and then carried out an examination of the former position. A compromise approach is advanced by Acquaviva in Chapter 3 . Under this system, the entity nominal the boys, for instance, is assigned a

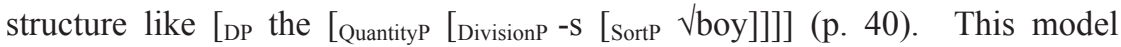
is like DM in leaving roots category-less, allowing the category "Sort" for

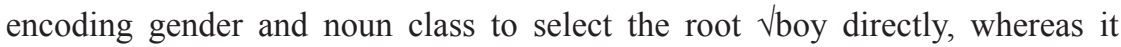
is unlike the recent version of DM in disallowing the direct merge of a root and a categorizing head. Thus, along with the CC framework, it has no need for zero categories. Consequently, this approach avoids the problems described above for zero morphemes, although it may still entail the same type of questions presented above for vocabulary entries and insertion.

\subsection{Relation between Root and Phase}

\subsubsection{Root and Phase Head}

The fourth major theme of the volume is a consideration of the interrelationships between root and phase. Root and phase are closely related to each other; phase can be partly defined in terms of root and hence the scope of phase depends on the definition of root. It is also noteworthy that the interaction between root and phase is affected by the kind of phase head. In-depth analyses of this root-phase relationship are presented by Gallego in Chapter 9 and by Lowenstamm in Chapter 11. Let us begin by examining the arguments put forward in Chapter 9.

The point of Chapter 9 is that argument structure is determined by phase head, and not by root. Among the phase heads, a light verb $(v)$ exclusively determines the argument structure, since only light verbs have unvalued $\varphi$ features and the roots governed by the features can take arguments. That the features in question are exclusively contained in $v$ s implies that nominals take no arguments. Although it is highly controversial whether nominals have arguments or not, the chapter advocates and justifies the latter position. What should be noted here about roots is that roots themselves lack syntactic arguments, though they contain conceptual content or "conceptual arguments." It should also be noticed that phase heads are classified into two types: case-assigning heads $(v, C)$ and categorizing heads $(x$ heads such as $n, v$, and $a$ ). In their relation to roots, $x$ heads only categorize roots, whereas light verb $(v)$ and $\mathrm{C}$ have a greater influence on roots, assigning them not only categories but also cases (and arguments). 


\subsubsection{Root and Phase Domain}

Chapter 11 discusses how stress shift in multiple suffixation is not fully accounted for within the localist theory of DM. In particular, the contrast between atomícity and atómicness is not explained, because the non-distinction of level $1 / 2$ affixes places both -ity and -ness in the same structural position. It is proposed then that affixes are roots and level 1 and level 2 "affixes" (bound roots) have the uninterpretable features of $[\mathrm{u} \downarrow]$ and $[\mathrm{uxP}]$ respectively. Thus, atomicitiless has the structure: $\left[{ }_{a p} a\left[v_{\mathrm{p}} \sqrt{l} \operatorname{less}_{[\mathrm{uxP}]}\left[{ }_{n \mathrm{p}} n\right.\right.\right.$

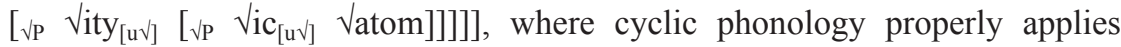
only in the first phase, the $n$-headed phase.

Although a correlation between affix classification and its ordering has been observed (Allen (1978)), it has not been seriously disputed in the DM literature, partly because the lexicon as a generative mechanism does not exist in this scheme. The present work deserves recognition for the redefinition of the correlation within the DM framework; the difference of level 1 and 2 affixes in stress-neutrality described above and the prohibition of level 1 affixes from occurring outside level 2 (e.g. *skillessity) originate in their occurrence in different syntactic loci. ${ }^{6}$ Furthermore, the semantic contrast between these two classes mentioned in 2.2 stems directly from their syntactic configurations. Note that the scope of phase in this system becomes widened: no level 1 "affixes," except the outermost one, constitute a phase, so that spheroidicity is made up of one phase. This has the merit of enclosing non-compositional meanings in the local domain of phase 1. This thus enables us to provide part of the solution to the problems for the Marantz/Arad hypothesis which were discussed in 2.2. There remain two specific questions and one fundamental question, though: (i) how does this system cope with the "ordering paradox," where a level 1 affix appears outside a level 2 affix (cf. unworkability) (Selkirk (1982: 100-106))?, (ii) certain level 2 suffixes have an affinity for other suffixes-notably, -ness preferentially joins to -ous base forms as in mysteriousness (Aronoff (1976: 45)) - so how are they linked together, given the undesirable intervention of an $x$ head between them?, and (iii) exactly what definition is given to the term "root," which is usually defined as a bound morpheme that becomes the core of a word?

${ }^{6}$ To obtain the right result, an assumption is necessary: categories head roots, not vice versa (p. 244). 


\section{Conclusion}

The book under review is an important contribution both to bringing out the theoretical significance of root investigation and providing considerable incentives for future exploration. It presents different views of roots focusing on four major issues in generative grammar: the categorization of category-neutral roots and their roles in sentence construction, idiosyncrasy treatment, and phase formation. The identification of roots as bound morphemes opens up new avenues for the proper construction of argument structures, the precise location of idiosyncrasies, and the adequate handling of phonological and morphological regularities. More generally, it will spark further reflection about the important issues of vocabulary insertion, functional categories within words, and phase-based word construction. What particularly remains to be explored is when and how to introduce roots into terminal nodes and how best to deal with null categorizers.

\section{REFERENCES}

Allen, Margaret Reece (1978) Morphological Investigations, Doctoral dissertation, University of Connecticut.

Aronoff, Mark (1976) Word Formation in Generative Grammar, MIT Press, Cambridge, MA.

Embick, David (2010) Localism versus Globalism in Morphology and Phonology, MIT Press, Cambridge, MA.

Halle, Morris (1994) "Distributed Morphology and the Inflection of Words," ms., MIT.

Harley, Heidi and Rolf Noyer (2000) "Formal versus Encyclopedic Properties of Vocabulary: Evidence from Nominalisations," The Lexicon-Encyclopedia Interface, ed. by Bert Peeters, 349-374, Elsevier, Amsterdam.

Marantz, Alec (1997) "No Escape from Syntax: Don't Try Morphological Analysis in the Privacy of Your Own Lexicon," University of Pennsylvania Working Papers in Linguistics 4, 201-225.

Marantz, Alec (2013) "Locality Domains for Contextual Allomorphy across the Interfaces," Distributed Morphology Today: Morphemes for Morris Halle, ed. by Ora Matushansky and Alec Marantz, 95-115, MIT Press, Cambridge, MA.

Morita, Junya (2007) "Where Does Nominalization Take Place?-An Antilexicalism Model," JELS 24, 141-150.

Nida, Eugene A. (1949) Morphology: The Descriptive Analysis of Words, University of Michigan Press, Ann Arbor.

Roeper, Thomas (1993) "Explicit Syntax in the Lexicon: The Representation of 
Nominalizations," Semantics and the Lexicon, ed. by James Pustejovsky, 185220, Kluwer, Dordrecht.

Selkirk, Elisabeth O. (1982) The Syntax of Words, MIT Press, Cambridge, MA.

[received July 8, 2016, revised and accepted February 2, 2017]

College of Humanities

Kinjo Gakuin University

2-1723 Omori Moriyama-ku, Nagoya

Aichi 463-8521

e-mail: morita@kinjo-u.ac.jp 